
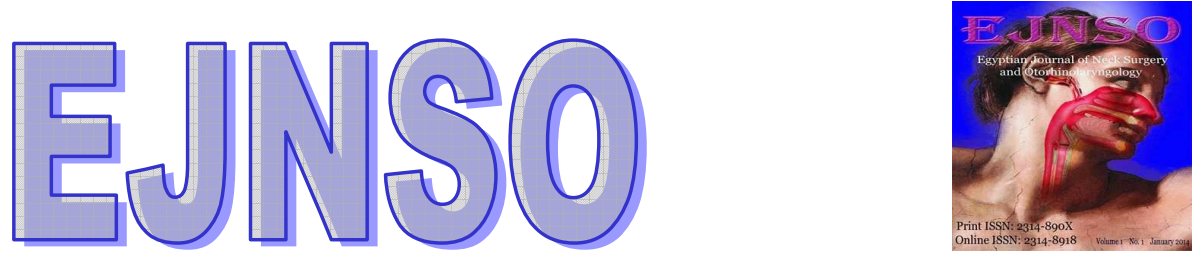

\title{
Diffusion-weighted Magnetic Resonance Imaging of Sinonasal Masses: Does Bone Erosion or Destruction Alter the Apparent Diffusion Coefficient Value in Differentiating Benign from Malignant Masses?
}

\author{
Osman, M.M ${ }^{\text {a }}$, Soliman, R. K., and Imam, H. M. K. ${ }^{\text {b }}$ \\ ${ }^{a}$ Otolaryngology Department, Faculty of Medicine, Assiut University, Assiut, Egypt. \\ ${ }^{\mathrm{b}}$ Department of Diagnostic Radiology, Faculty of Medicine, Assiut University, Egypt
}

Received: February $11^{\text {th }}, 2015$.

Revised: March $21^{\text {st }}, 2015$.

Accepted: April $7^{\text {th }}, 2015$

Keywords: diffusion weighted MRI, sinonasal masses, bone erosion, destruction

Abbreviations: DWI: diffusion weighted imaging, cMRI: conventional magnetic resonance imaging, CT:computed tomography, ROI: regions of interest, ADC: apparent diffusion coefficient.

\section{ABSTRACT}

Objective: Detect whether bone erosion or destruction alters the ADC value while discriminating benign from malignant sinonasal masses.

Patients and methods: Twenty five patients were investigated and operated upon in this study. Preoperative CT scans and diffusion weighted MRI imaging were done within 48 hours prior to surgery. Surgical procedures were done in the form of complete resection or biopsy taking. The ADC values were calculated for sinonasal lesions associated with bone erosion or destruction detected on CT scanning.

Results: Histopathological diagnosis revealed benign masses in $44 \%$ and malignant tumors in $56 \%$ of biopsies. No significant differences between mean ADC values of the first and second ROI in both benign $(\mathrm{P}$ value $=0.365)$ and malignant masses $(\mathrm{P}$ value $=0$. 07). Statistical significant difference was found between the ADCL values of benign and malignant sinonasal masses. $(\mathrm{P}$ value $=0.044)$. Conclusions:

Bone erosion or destruction does not alter the ADC value in differentiating between benign and malignant masses. ADC value does not change with bone erosion or destruction.

\section{Introduction:}

Development of the technique of MRI has led to the use of diffusionweighted imaging in screening of various body parts and diagnosing different lesions [1-4]. A great benefit of DWI in diagnosing neoplasms is that DWI reveals the biological character of the tissue [5]. Sinonasal tumors are of low incidence. However, they typically have poor prognosis owing to their early extension to the surrounding structures, most importantly, intracranial extension [6]. A wide variety of both benign and malignant sinonasal tumors have been recorded. Differentiation between benign and malignant tumors is essential for the treatment plan. CT and conventional MRI (cMRI) are mainly used to diagnose sinonasal tumors. MRI is more useful for soft tissue characterization, whereas CT is better to assess bone involvement. Both provide useful information about tumor extension but they are lacking sensitivity and accuracy as they depend on volumetric and morphological criteria $[7,8]$. So, it is not always easy to differentiate between benign and 
malignant lesions [9-11]. Diffusionweighted imaging (DWI) and ADC value are used to reflect tissue cellularity. Therefore, they were found to be useful in discrimination between benign and malignant neoplasms [12-15]. Many authors reported that the mean $\mathrm{ADC}$ value of benign solid lesions was significantly higher than that of malignant tumors $[12,16]$. In sinonasal tumors, however, bone erosion and destruction are associated not only with malignant lesions but also with some benign lesions [17]. Therefore, this study was designed to identify whether bone erosion or destruction alters the ADC value while discriminating benign from malignant sinonasal masses.

\section{Materials and Methods:}

\section{Patients:}

Patients presented clinically with sinonasal tumors were scanned using low dose multidetector CT. Only patients having sinonasal mass with bone erosion or destruction were included. Therefore a total number of 25 patients (17 males and 8 females) were enrolled in this study.

\section{CT protocol:}

Patients were scanned using spiral 16 multi-detectors CT in axial section from the top of the frontal sinus till the end of the hard palate. Parameters were used: 120 $\mathrm{KV}, 40 \mathrm{mAs}, 2.5 \mathrm{~mm}$ collimation, 3- $\mathrm{mm}$ slice thickness, $1.2-\mathrm{mm}$ reconstruction increment, and a pitch of 1 .

\section{MRI protocol:}

Patients were scanned using 1.5 Tesla MRI scanner. They were examined in supine position with standard polarized head coil. Routine MRI was performed using the following sequences: Spin echo axial T1-weighted imaging (TE/ TR: 650/12 ms), axial and coronal T2weighted imaging (TE/TR: 4800/98 ms) with slice thickness of $5 \mathrm{~mm}$ and interslice gap of $1.5 \mathrm{~m}$. The field of view (FOV) used was 190 x $190 \mathrm{~mm}$ and matrix size of $320 \times$ x 320. After intravenous administration of Gadolinium -DPTA $(\mathrm{Gd})$, contrast enhanced T1WI in axial and coronal planes were obtained in 21 patients. DWI was then performed on axial scans using single shot echo planner spin echo (EPI). The following parameters were used: (TE/TR: 6800/98 ms), with slice thickness of $5 \mathrm{~mm}$ and inter-slice gap of $1.5 \mathrm{~mm}$. The field of view (FOV) used was $250 \times 250 \mathrm{~mm}$ and matrix size of 192 x 192.

Diffusion gradient encoding was performed in three orthogonal planes (X, Y, Z). Three $b$ - factors (0-400-800) were obtained. ADC maps were then generated. In each patients, two regions of interest (ROI) were selected, the first ROI was drawn at the area of bone erosion or destruction, whereas the second was drawn at the area recorded the lowest $\mathrm{ADC}$ value in the lesion, away from the area of bone destruction. For each patient, the lowest ADC reading, whether that of the first or the second ROI has been selected to represent the mean ADC of the lesion (ADCL).

\section{Histopathological data:}

Histopathological diagnosis was obtained either by biopsy or after surgical resection.

\section{Statistical analysis:}

Data analysis was performed using SPSS statistical software package. Nonparametric Mann- Whitney U tests were used to evaluate the statistical difference between ADCL values of benign and malignant masses. Then, for each of benign and malignant masses, statistical differences between the ADC values of the selected two ROIs were calculated. Pvalues less than 0.05 were considered significant.

\section{Results:}

Our study populations consisted of 25 patients (17 males and 8 females). Age ranged from 13 to 58 years. On the basis of histopathological diagnosis, sinonasal masses were divided into benign masses $44 \%$ and malignant tumors $56 \%$. The 
distribution of pathology is shown in (Table 1)

For both benign and malignant lesions: The mean ADCL value of benign sinonasal lesion was $1.14 \pm 0.41 \times 10^{-3}$ $\mathrm{mm}^{2} / \mathrm{s}$, whereas the mean ADC of malignant sinonasal lesion was $0.87 \pm 0.31$ $\times 10^{-3} \mathrm{~mm}^{2} / \mathrm{s}$. Statistically significant difference was found between the ADCL values of benign and malignant sinonasal masses. $(\mathrm{P}$ value $=0.044)($ Figure: 1$)$.

In benign sinonasal masses:

The mean ADC value of the first ROI was $1.18 \pm 0.45 \times 10^{-3} \mathrm{~mm}^{2} / \mathrm{s}$., whereas the mean ADC of the second ROI was $1.35 \pm$ $0.43 \times 10^{-3} \mathrm{~mm}^{2} / \mathrm{s}$. No significant differences were found between mean ADC values of the first and second ROI (P value $=0.365$ ). (Figure: 2 ).

\section{In Malignant sinonasal tumors:}

The mean ADC value of the first ROI was $0.98 \pm 0.36 \times 10^{-3} \mathrm{~mm}^{2} / \mathrm{s}$, whereas the mean ADC of the second ROI was $1.22 \pm$ $0.35 \times 10^{-3} \mathrm{~mm}^{2} / \mathrm{s}$. No significant differences between mean ADC values of the first and second ROI were found $(\mathrm{P}$ value $=0.07$ ) (Figure: 3 ).

Table (1): showing the final histopathological diagnosis of 25 patients.

\begin{tabular}{|l|c|c|}
\hline Pathology & Number & \\
\hline Inflammatory polyps & 4 & \multirow{2}{*}{ Benign } \\
\cline { 1 - 2 } Inverted papilloma & 2 & \\
\hline Mucocele & 3 & \\
\hline Juvenile psammomatoid ossifying fibroma & 1 & \multirow{2}{*}{ Malignant } \\
\hline Juveniles angiofibroma & 1 & \\
\hline Squamous cell carcinoma & 9 & \\
\hline Non - Hodgkin lymphoma & 3 & \\
\hline Olfactory neuroblastoma & 1 & \\
\hline Adenocarcinoma & 1 & \\
\hline
\end{tabular}

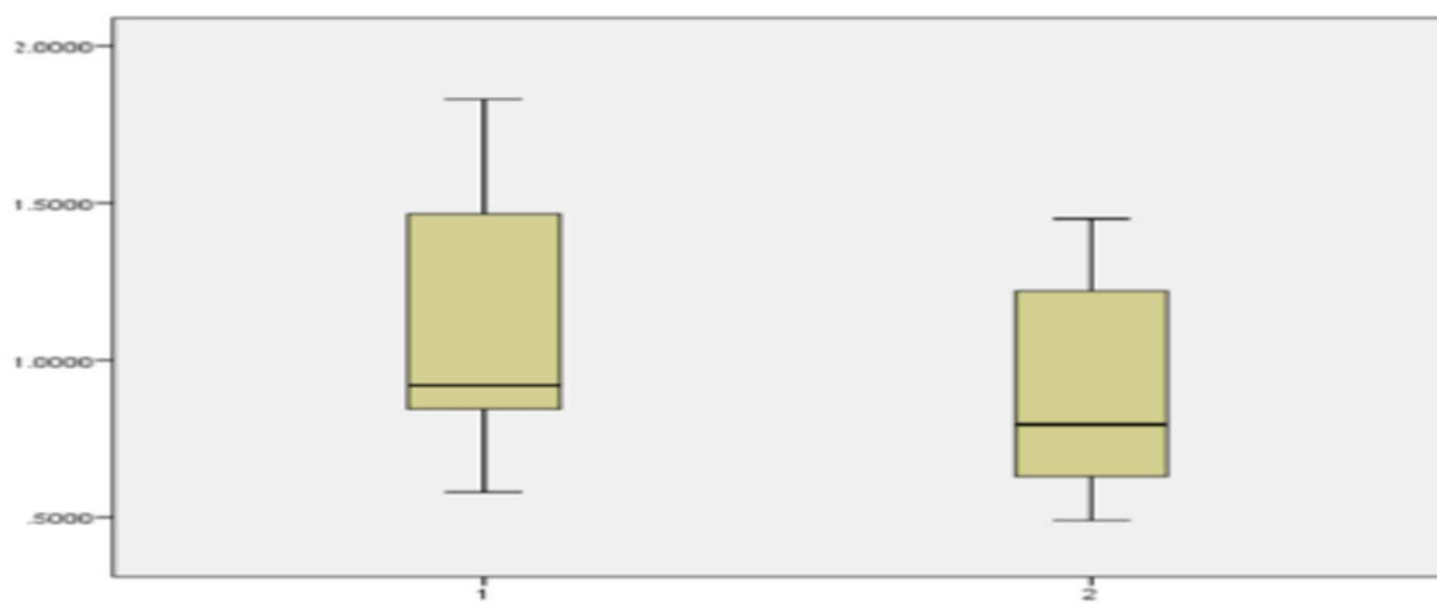

Fig. 1: Box plots comparing the mean ADCL values of 1) benign and 2) malignant sinonasal tumors. The horizontal thick line is the median, and the vertical lines show the full range of values in the data. ADCL of malignant neoplasm was significantly lower than that of benign lesions. $(\mathrm{P}$ value $=0.044)$. 


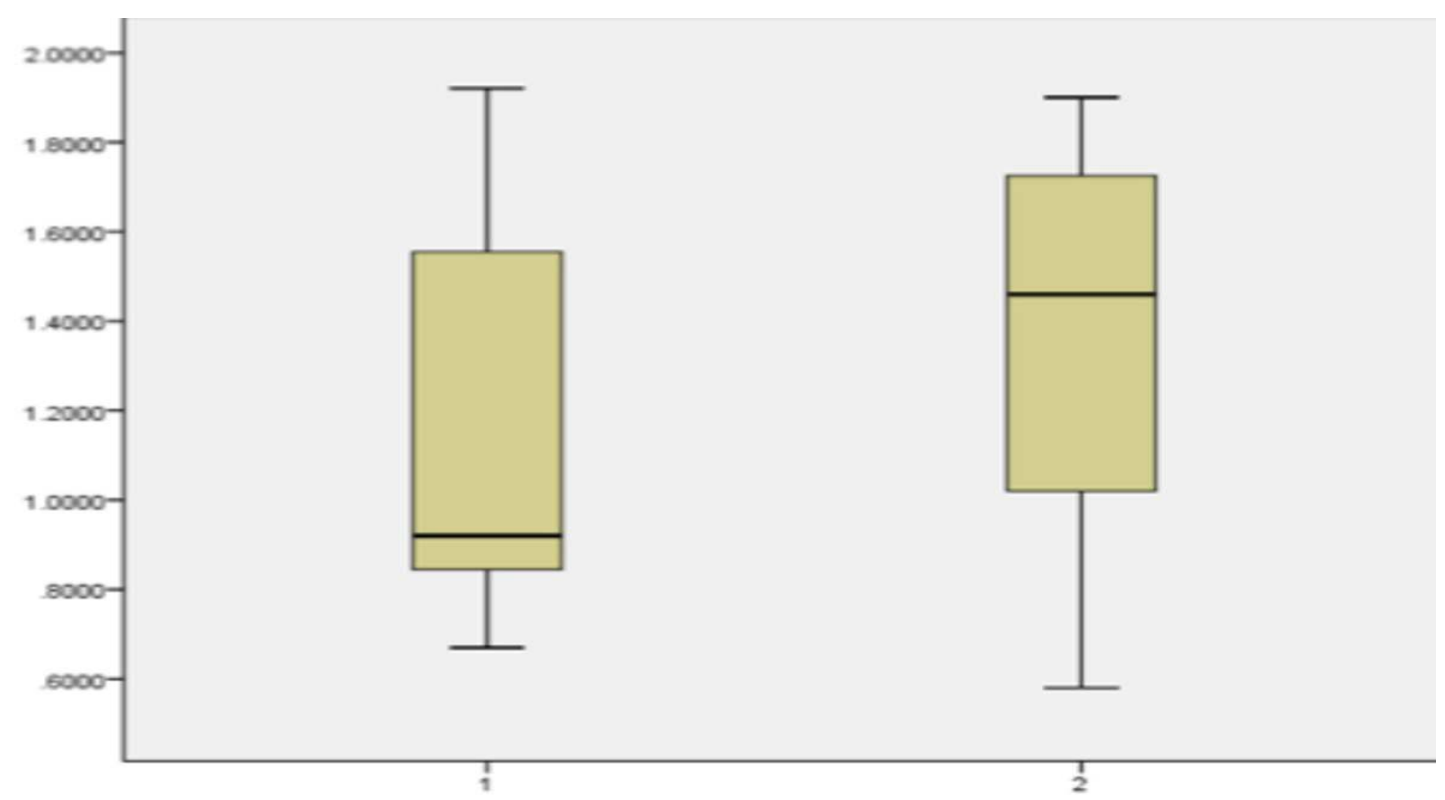

Fig. 2: Box plots comparing the mean ADC values of 1) the first ROI and 2) the second ROI of benign sinonasal tumors. The horizontal thick line is the median, and the vertical lines show the full range of values in the data. No significant difference was found between the $\mathrm{ADC}$ values of the two ROIs.

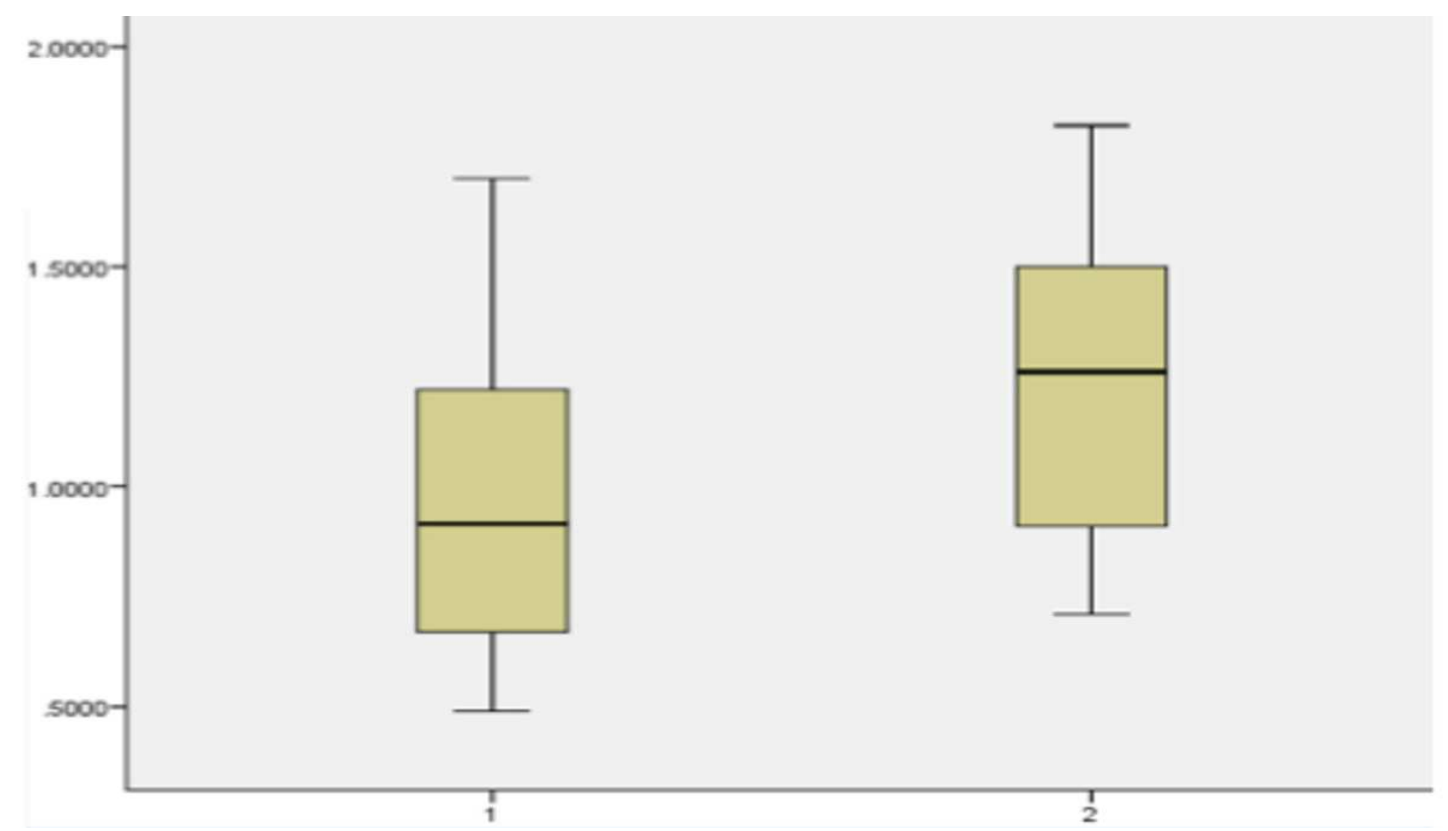

Fig. 3: Box plots comparing the mean ADC values of 1) the first ROI and 2) the second ROI of malignant sinonasal tumors. The horizontal thick line is the median, and the vertical lines show the full range of values in the data. No significant difference was found between the ADC values of the two ROIs. 


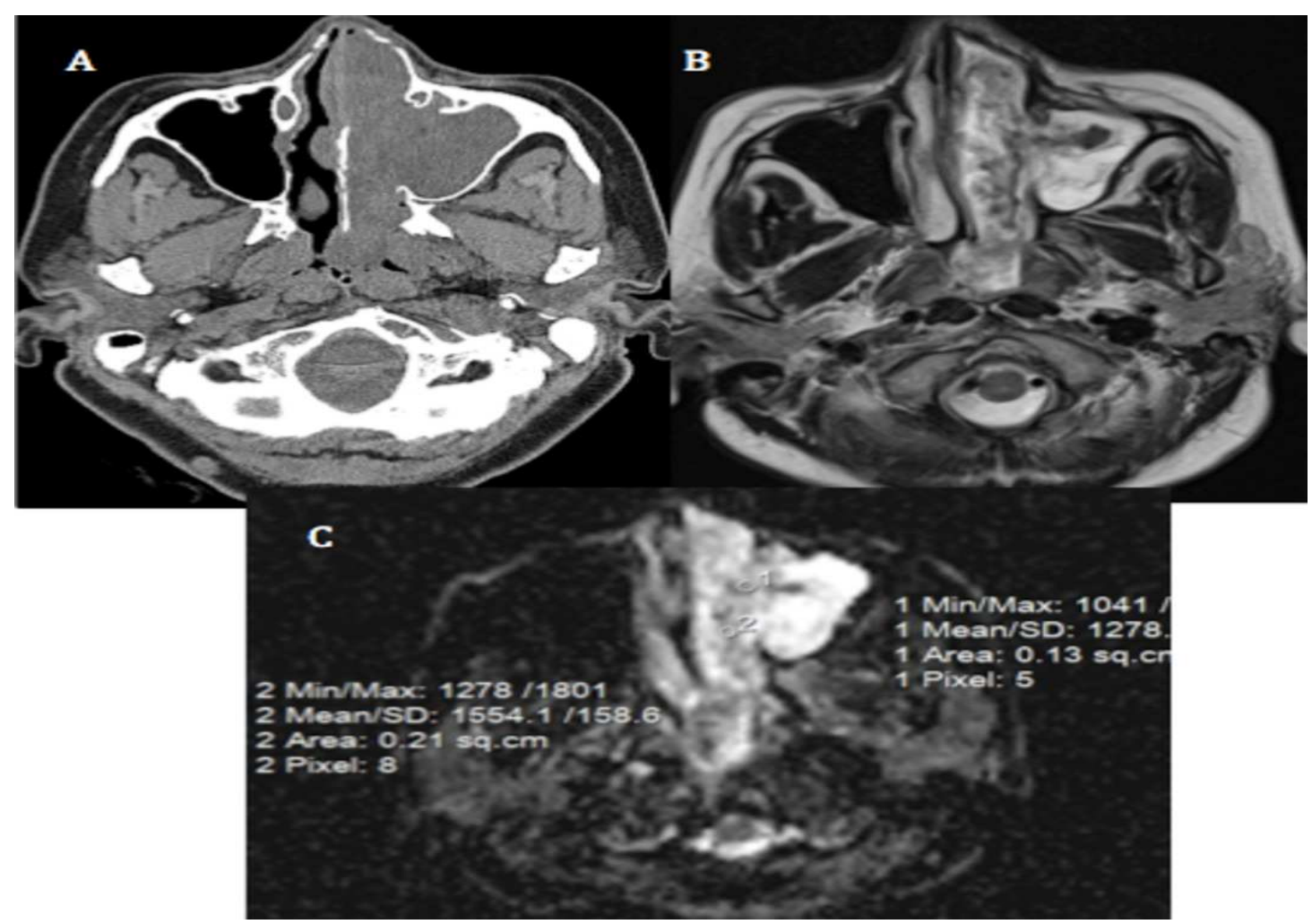

Fig. 4: Inflammatory polyp. A) Axial CT shows polypoidal thickening involving the left nasal cavity and left maxillary sinus associated with bone remolding of the upper nasal septum and left maxillary sinus. B) Axial T2WI shows polypoidal thickening in the left nasal cavity extending to the left maxillary sinus with retained maxillary secretion, eliciting high SI. C) Axial ADC map shows the ADC value of the first ROI (at the area of bone remolding) $=1.2 \times 10-3 \mathrm{~mm} 2 / \mathrm{s}$, whereas the mean $\mathrm{ADC}$ value of the second $\mathrm{ROI}=1.510-3 \mathrm{~mm} 2 / \mathrm{s}$.

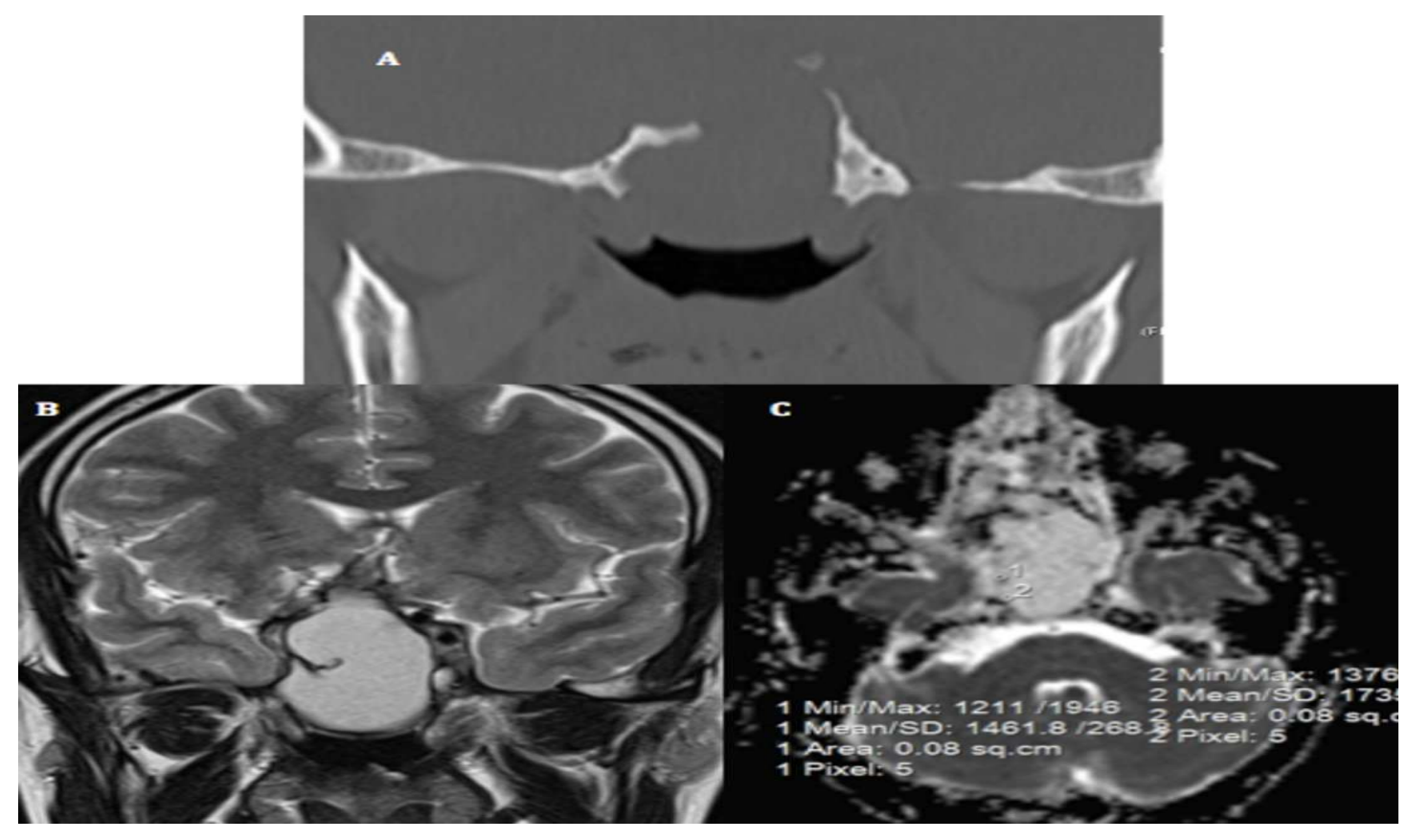

Fig. 5: Sphenoid sinus mucocele. A) Coronal CT and B) Coronal T2WI show large mucocele filling and expanding the sphenoid sinus associated with destruction of the sphenoidal sinus wall. C) Axial ADC map shows the mean ADC value of the first $\mathrm{ROI}=1.4 \times 10-3 \mathrm{~mm} 2 / \mathrm{s}$, whereas the mean ADC value of the second $\mathrm{ROI}=1.710^{-3} \mathrm{~mm}^{2} / \mathrm{s}$. 


\section{Discussion:}

It is possible to observe similar radiological findings for benign and malignant sinonasal lesions as some benign tumors extend into surrounding structures while some malignant tumors are slowly growing. Therefore; their distinction may be difficult and unreliable as their appearance using CT and cMRI of these lesions is not pathognomonic and distinction is necessary for detecting prognosis and planning treatment $[6,9,10 \& 18-22]$.

Magnetic resonance imaging is a fundamental tool in the diagnosis of sinonasal lesions as it distinguishes normal and inflamed soft tissues and differentiates between these tissues and tumor better than computed tomography. MRI is often used in combination with $\mathrm{CT}$ to precisely delineate the extent of these neoplasms [23]. Involvement of the skull base, the orbits, the intracranial compartment, and potential perineural spread of tumor can influence treatment options. For this reason, Magnetic resonance is essential to evaluate these tumors as they are often advanced and difficult to treat at the time of diagnosis because of the complex anatomy of the sinonasal region and its proximity to the previously mentioned critical structures [24].

The biophysical mechanism of DWI is based on the microscopic random translational motion of water molecules in biological tissues. The magnitude of this motion is characterized by its apparent diffusion coefficient (ADC) values. Variation in ADC values reflects the alteration and redistribution of water molecules between intracellular and extracellular compartments of a tissue [25]

In the current study, the mean ADC value of malignant sinonasal lesions was significantly lower than that of benign lesions $(\mathrm{P}=0.04)$. The lower ADC value of the malignant tumors is mainly due to high tumor cellularity and reduced water content in the interstitial space $[12,15,26]$. In agreement with our results, Razek et al. [11] reported a significant difference between mean ADC values of benign and malignant paranasal masses. They further found a significant difference between $\mathrm{ADC}$ values of different grades of malignant tumors.

Malignant lesions of the nasal cavity are rare, but the similar clinical features of the benign and malignant lesions in the beginning may delay the diagnosis. [27]. In sinonasal imaging, the general rules are that benign tumours cause remodelling and thickening of adjacent bone, while malignant tumours destroy the bone [28]. Inflammatory diseases could cause destruction and erosions of the organ that resemble that caused by malignant lesions and may be misdiagnosed. [27,29].

On the other hand, some malignant tumours remodel bones rather than destroy it; e.g. sinonasal sarcomas, minor salivary gland carcinomas, extramedullary plasmacytomas, large cell lymphomas, olfactory neuroblastomas and hemangiopericytomas [28].

Our study revealed that, bone erosion or destruction does not alter the ADC value in differentiating between benign and malignant masses. As there were no significant difference between the mean ADC values between the first and the second ROI in both benign and malignant masses.

\section{Conclusion}

$\mathrm{ADC}$ value is a useful quantitative parameter to differentiate between benign and malignant sinonasal masses and its value does not change with bone erosion or destruction.

\section{References}

1. Filippi $M$, Cercignani $M$, Inglese $M$, Horsfield MA, Comi G. 2001. Diffusion tensor magnetic resonance imaging in multiple sclerosis. Neurology 56: 304-311

2. Bammer R. 2002. Basic principles of diffusion-weighted imaging. Eur $\mathrm{J}$ radiol 45: 169-184

3. Guo Y, Cai YQ, Cai ZL, Gao YG, An NY, Ma L, Mahankali S, Gao JH. 2002. Differentiation of clinically benign and malignant breast lesions using diffusion- 
weighted imaging. J Mag Reson Imaging 16: 172-178

4. Lyng H, Haraldseth O, Rofsrad EK. 2000. Measurement of cell density and necrotic fraction of human melanoma xenografts by diffusion-weighted magnetic resonance imaging. Magn Reson Med 43: 828-836

5. Woodhams R, Matsunaga K, Kan S, Hata $\mathrm{H}$, Ozaki M, Iwabuchi K, Kuranami M, Watanabe M, Hayakawa K. 2005. ADC Mapping of Benign and Malignant Breast Tumors. Magn Reson Med Sci. 4(1):35-42.

6. Loevner LA, Sonners AI. 2004. Imaging of neoplasms of the paranasal

sinuses. Neuroimaging Clin N Am 14:625-46

7. Imaizumi A, Yoshino N, Yamada I, Nagumo K, Amagasa T, Omura K, Okada N, Kurabayashi T. 2006. A potential pitfall of MR imaging for assessing mandibular invasion of squamous cell carcinoma in the oral cavity. AJNR Am J Neuroradiol 27:114122.

8. Ahmad A, Branstetter BFT. 2008. CT versus MR: still a tough decision. Otolaryngol Clin North Am 41:1-22.

9. Maroldi R, Ravanelli M, Borghesi A, Farina D. 2008. Paranasal sinus imaging. EurJ Radiol 66:372-86

10. Connor S, Hussain S, Woo E. 2007. Sinonasal imaging. Imaging 19:39-30

11. Razek AA, Sieza S, Maha B. 2009. Assessment of nasal and paranasal

sinus masses by diffusion-weighted MR imaging. J Neuroradiol 36:206-11

12. Wang J, Takashima S, Takayama F, Kawakami S, Saito A, Matsushita T, Momose M, Ishiyama T. 2001. Head and neck lesions: characterization with diffusion-weighted echo-planar MR imaging. Radiology 220:62130

13. Kitis O, Altay H, Calli C, Yunten N, Akalin T, Yurtseven T. 2005. Minimum apparent diffusion coefficients in the evaluation of brain tumors. Eur $\mathbf{J}$ Radiol 55:393-400

14. Yamasaki F, Kurisu K, Satoh K, Arita K, Sugiyama K, Ohtaki M, Takaba J, Tominaga A, Hanaya R, Yoshioka H, Hama S, Ito Y, Kajiwara Y, Yahara K, Saito T,Thohar MA . 2005. Apparent diffusion coefficient of human brain tumors at MR imaging. Radiology 235:985-91

15. Lee EJ, Lee SK, Agid R, Bae JM, Keller A, Terbrugge K. 2008. Preoperative grading of presumptive low-grade astrocytomas on MR imaging: diagnostic value of minimum apparent diffusion coefficient. AJNR Am J Neuroradiol 29:1872-7

16. Chawla S, Kim S, Wang S, Poptani H. 2009. Diffusion-weighted imaging in head and neck cancers. Future Oncol. 5(7):959-75. 17. Kim HJ, Kim JH, Kim JH, Hwang EG. 1995.

Bone erosion caused by sinonasal cavernous hemangioma: CT findings in two patients. AJNR Am J Neuroradiol. 16 (5):1176-1178.

18. Rao VM, El-Noueman KI. 1998. Sinonasal imaging. Anatomy and pathology.Radiol Clin North Amer 36:921- 39 19. Hudgins PA. 1996. Sinonasal imaging. Neuroimag Clin North Amer 319-30

20. Allbery SM, Chal jub G, Cho N, Rassekh CH, John SD, Guinto FC. 1995. MR imaging of nasal masses. RadioGraphics 15:1311-27

21. Branstetter B, Weissman J. 2005. Role of $\mathrm{MR}$ and $\mathrm{CT}$ in the paranasal sinuses. Otolaryngol Clin North Amer 38:1279-99

22. Valencia M, Castillo M. 2008. Congenital and acquired lesions of the nasal septum: a practical guide for differential diagnosis. RadioGraphics 28:205-23

23. Som PM, Dillon WP, Curtin HD, Fullerton GD, Lidov M. 1990. Hypointense paranasal sinus foci. Differential diagnosis with MR imaging and relation to CT findings. Radiology 176:777-781.

24. Raghavan P, Phillips CD. 2007. Magnetic resonance imaging of sinonasal malignancies. Top Magn Reson Imaging 18(4):259-67.

25. Padhani AR, Liu G, Mu-Koh D, Chenevert TL, Thoeny HC, Takahara T, DzikJurasz A, Ross BD, Van Cauteren M, Collins D, Hammoud DA, Rustin GJS, Taouli B, Choyke PL 2009. Diffusion-weighted magnetic resonance imaging as a cancer biomarker: consensus and recommendations. Neoplasia 11:102-125.

26. Maeda M, Maier SE. 2008. Usefulness of diffusion-weighted imaging and 
the apparent diffusion coefficient in the assessment of head and neck tumors. J Neuroradiol 35:71-8

27. Som PM, Curtin HD 1994. Inflammatory Lesions and Tumors of the Nasal Cavities and Paranasal Sinuses with Skull Base Involvement. Neuroimaging Clinics of North American 4(3): 499-513.
28. Eggesb $\varnothing \quad \mathrm{HB}$ Imaging of sinonasal tumours. Cancer Imaging. 7(12): 136-152.

29. Murphey MD, Walker EA, Wilson AJ, Kransdorf MJ, Temple HT, Gannon FH 2003. Imaging of Primary Chondrosarcoma: Radiologic-Pathologic Correlation. RadioGraphics. 23 (5): 1245-1278. 